\title{
57. NOTAS COROLÓGICAS PARA LA FLORA DE ANDALUCÍA
}

\section{Ángel LORA GONZÁLEZ y José Luis VIVERO POL}

Chorological notes about the Andalusian Flora.

Palabras clave. Corología, ecología, Andalucía, España.

Key words. Chorology, ecology, Andalusia, Spain.

Con motivo de las diferentes campañas de colecta efectuadas desde el Jardín Botánico de Córdoba en virtud del desarrollo de distintos programas de investigación, pero muy especialmente en las realizadas con objeto de cumplimentar los Planes de Recuperación de diferentes especies vegetales encargados por la Agencia de Medio Ambiente de la Junta de Andalucía en los últimos años, se han podido incluir en el Herbario COA de la Unidad Docente de Botánica Agrícola y Forestal del Departamento de Ciencias y Recursos Agrícolas y Forestales de la E.T.S.I.A.M. de la Universidad de Córdoba unos 5.000 pliegos procedentes de toda la Comunidad Andaluza. Aportamos en esta nota 24 citas corológicas interesantes para el citado territorio.

Rupicapnos africana (Lam.) Pomel subsp. decipiens (Pugsley) Maire

CÁDIZ: Sierra de Grazalema, Puerto de las Palomas. 30STF8774. 1200 m. 16-VII-97. Lora y Vivero. COA 24269.

También se ha observado en los cortados dolomíticos situados sobre el arroyo de la Garganta Verde (30STF8577), sin pliego. Se trata pues de nuevas citas para el Parque Natural de Grazalema que completan las recogidas anteriormente por Aparicio y Silvestre (1987).

Arenaria armerina Bory subsp. caesia (Boiss.) C.

Díaz, C. Morales y F. Valle
MÁlAGA: Sierra Almijara, subida al puerto de Cómpeta. 30SVF2282. 19-VIII-90. Hernández Bermejo y Clemente. COA 18585.

Las localidades de este taxón en la provincia de Málaga son muy escasas, pues apenas existen citas y López-González (1990) la indica sin pliego de referencia.

\section{Silene oropediurum Cosson ex Batt.}

GRANADA: Fuente Nueva. 30SWG5275. 980 m. 24-V-96. Vivero y Prados. COA 24267.

Especie reciente para la flora europea (Peris \& Stübing, 1985), que se encontró por primera vez en Albacete y posteriormente en Alicante, Madrid y Almería (Talavera, 1990). En este caso ocupa un afloramiento calizo, con muy escaso suelo, rodeado por cultivos sobre suelos margo-yesosos. Es la primera cita para la provincia.

Malva neglecta Wallr.

JAÉN: Sierra de Mágina, Pico Almadén. 30SVG5376. 1950 m. 3-VIII-96. Vivero y Jiménez. COA 20897.

Aunque ya había sido citada en Mágina, su presencia no se constataba desde 1925 (Cuatrecasas, 1929). En el resto de la provincia es muy escasa (García-Hernández y Fernández-López, 1986), apareciendo sólo en Valdepeñas de Jaén (JAÉN 811865) y Cazorla (Benavente, 1996). También es escasa en Andalucía Occidental, ya que sólo está citada en la comarca del Valle de los Pedroches y en Cabra (García-Montoya y Muñoz, 1990).

Helianthemum pannosum Boiss. subsp. pannosum

Trabajo parcialmente elaborado en desarrollo de los Acuerdos Específicos VII y X de la Agencia de Medio Ambiente (Junta de Andalucía) y el Jardín Botánico de Córdoba sobre Planes de Recuperación de Especies Amenazadas 
JAÉN: Sierra de Mágina, Barranco de la Tosquilla. 30SVG5873. 1500 m. 1-VIII-96. Vivero y Jiménez. COA 20893.

Endemismo estricto de Sierra Nevada (Cerro del Trevenque, 1500-1900 m) que presenta aquí su primera cita provincial y una ampliación notable de su área de distribución.

\section{Sisymbrium macroloma Pomel}

JAÉN: Sierra de Segura, Cueva del Agua. 30SWH3221. 1280 m. 23-VIII-97. Vivero y Benavente. COA 24293.

Parece ser la primera localidad confirmada para Andalucía, ya que Schulz (1957) señala su presencia en la provincia de Málaga (Gobantes y Yunquera) basándose en citas de Rouy, pero no existe pliego de herbario que lo confirme. Ocupa un hábitat (cuevas con sustrato nitrificado por el ganado) descrito como típico para otras localidades de la Península (Escudero et al., 1989).

\section{Hormatophylla lapeyrousiana (Jordan) Küpfer} subsp. angustifolia (Willk.) Rivas Mart.

JAÉN: Sierra de Mágina, Barranco de la Tosquilla. 30SVG5874. 1520 m. 1-VIII-96. Vivero y Jiménez. COA 20895.

La primera y única cita para Mágina se publicó recientemente (Blanca, 1989), con lo que ésta confirma la presencia del taxón en la sierra. También aparece en las sierras de Cazorla y Segura (Benavente, 1996) y en Los Villares (Medina y Fernández-López, 1988), en todos los casos en grietas de rocas calizas.

Draba hispanica Boiss. subsp. laderoi Rivas Mart.,

M.E. García y Penas

ALMERÍA: Pico del Almirez. 30SWG0704. 2500 m. 17-VI-94. Pujadas y Pallarés. COA 22924.

Esta especie fue descrita sobre material del Puerto de la Ragua, Granada (Rivas-Martínez et al., 1991) y se considera un endemismo nevadense; en Blanca (1993) aparece también citada para el área almeriense de Sierra Nevada. Se trata pues, de la segunda cita para la provincia de Almería, que amplía su distribución hacia el E, alejándose del núcleo central silíceo nevadense, único enclave descrito hasta ahora para el taxón.

Iberis grosii Pau

MÁLAGA: Sierra Blanca, Ojén, cerca de la carretera de Monda, al borde de una cantera. 30SUF3451. 500 m. 21-VII-93. Lora, Hernández Bermejo y Martín-Consuegra. COA 20913.

Esta especie es una de las menos herborizadas de España (Moreno, 1983). Se trata de un endemismo exclusivo de las Sierras Tejeda y Almijara, para el que esta localidad representa el extremo occidental de su área.

Coincya monensis (L.) Greuter \& Burdet subsp. nevadensis (Willk.) Leadlay

JAÉN: Sierra Mágina. 19-IX-82. Montoro. COA 15405.

Parece ser la primera cita para Jaén de esta especie que se creía circunscrita al macizo nevadense, tanto en Almería como en Granada (Leadlay, 1993). Además se presenta por debajo de su rango altitudinal $(2300-3200 \mathrm{~m})$ ya que en la Sierra de Mágina sólo se alcanzan $2100 \mathrm{~m}$.

\section{Vella spinosa L.}

GRANADA: Venta Micena. 30SWG5378. 980 m. 24-V-96. Vivero y Prados. COA 24262.

Es la localidad más baja de la Península Ibérica, difiriendo en unos $300 \mathrm{~m}$ del rango mínimo (GómezCampo, 1993). Es un endemismo bético que caracteriza al matorral xeroacántico de alta montaña caliza. Sin embargo, aquí se presenta en un terreno llano, de carácter estepario, con suelos gípsicos. Hemos encontrado sólo dos individuos, uno de ellos de gran tamaño.

\section{Sorbus domestica L.}

JAÉN: Baños de la Encina, cerca del Centenillo, pico Montón de Trigo. 30SVH4349. 14VII-97. Lora y Moreno. COA 24265.

No conocemos ninguna cita de la especie para Sierra Morena ya que prefiere sustratos calizos, de ahí la importancia corológica y ecológica de esta localidad. Se descarta que proceda de asilvestramiento debido a su aislada situación pues se encuentra en un rebollar mixto y perfectamente conservado de Quercus pyrenaica acompañado de Quercus suber y Sorbus torminalis entre otros.

Prunus mahaleb L.

CÓRDOBA: Cabra, Sierras Subbéticas, poldjé de La Nava. 30SUG7950. IV-94. Castillo. COA 20582.

Primera cita para las Sierras Subbéticas y 
segunda provincial, pues ya había sido citada en la Sierra de Hornachuelos (Valdés et al., 1987).

\section{Prunus spinosa $\mathrm{L}$.}

GRANADA: Moclín, camino de Malalmuerzo. 30SVG2832. 10-II-1996. Arcos. COA 20740.

La primera cita provincial es reciente y se hizo para la Sierra de Alfácar (Valle y Díaz de la Guardia, 1987). Posteriormente aparecieron nuevas localidades: Cogollos-Vega, Iznalloz y Huéscar. A pesar de ser relativamente abundante en las Sierras Béticas apenas hay citas en la provincia. Algunos autores lo atribuyen a que es sustituida en la zona por Prunus ramburii, opinión que no compartimos en su totalidad ya que tienen preferencia por hábitats diferentes pues $P$. spinosa es propia del piso mesomediterráneo y $P$. ramburii del oromediterráneo.

\section{Prunus prostratus Labill. f. erecta Molero}

MÁLAGA: Sierra del Jobo, Pico del Chamizo. 30SUF8394. 1600 m. 10-VI-95. Vivero. COA 17545.

Fue citada hace 15 años en Serreta Negra de Fraga, Huesca, por Molero (1981) a muy baja altura $(300 \mathrm{~m})$ en el fondo de una vaguada con abundante humedad. Esta se encuentra en un ambiente distinto pues se presenta colgada de la pared de una profunda grieta, en la cima de la sierra.

\section{Erodium tordylioides (Desf.) L'Her.}

CÁDIZ: Zahara, en lo alto del promontorio del Castillo. 30STF8780. 550 m. 17-VII-97. Lora y Vivero. COA 24264.

Aparicio (1993) citaba esta localidad como la única para Andalucía de este taxón iberonorteafricano; posteriormente Albareal y Romero Zarco (1993) lo señalan también para la Sierra de la Peñagua (Sevilla). Hemos comprobado que la población de Cádiz se ha incrementado con varias plantas en la cima del promontorio, protegidas entre las rocas.

\section{Carum verticillatum (L.) Koch}

HUELVA: Parque Natural de Doñana, turbera del Abalario. 29SQB0812. 40 m. 20-VII-97. Lora y Vivero. COA 24263.

Su distribución se limitaba sólo a los límites de la Reserva Biológica de Doñana (FernándezGaliano y Cabezudo, 1976), por lo que se trata de la primera localidad fuera de la misma.

\section{Scutellaria minor Hudson}

HUELVA: Parque Natural de Doñana, turbera del Abalario. 29SQB0812. 40 m. 20-VII-97. Lora y Vivero. COA 24377.

Hasta ahora sólo había sido descrita dentro de los límites del Parque Nacional de Doñana (RivasMartínez et al., 1980). Esta nueva localidad amplía su área fuera del mismo.

\section{Campanula willkommii Witasek}

ALMERÍA: Base del Pico del Chullo. 30SWG0005. 2500 m. 17-VI-94. Pujadas y Pallarés. COA 18249.

Endemismo nevadense extendido por el núcleo central y con escasas citas en la zona almeriense. Se confirma la referencia aparecida en Sagredo (1987).

Leucanthemopsis pectinata (L.) G. López y Ch.E. Jarvis

ALMERÍA: Base del Pico del Chullo. 30SWG0005. 2400 m. 9-VI-94. Pujadas. COA 22923.

Esta es la segunda cita provincial, que confirma la de Sagredo (1987) en el Pico del Almirez.

\section{Leucanthemum aligulatum Vogt}

JAÉN: Sierra de Segura, arroyo del Tejuelo. 12-VII-97. Benavente y Nieto. COA 24294.

Se confirma la presencia en su única localidad de Andalucía, pues su última cita data de 1906 (Vogt, 1991). Su presencia es muy puntual, pues ya en esa fecha se citaba como rara en una sierra que, por otro lado, está muy prospectada.

\section{Hieracium elisaeanum Arvet-Touret}

JAÉN: Sierra de Mágina, vert. sur de Cerro Cárceles. 30SVG5777. 1940 m. 2-VIII-96. Vivero y Jiménez. COA 20892.

Aparece citada sólo en las Sierras de Cazorla, Segura y La Cabrilla (Hervier, 1905; FernándezGaliano y Heywood, 1960; Fernández-López, 1979; Alcántara et al., 1990 y Benavente, 1996), por lo que es la primera cita provincial fuera del Parque Natural.

Klasea flavescens (L.) Holub subsp. flavescens CÁDIZ: Sierra de Grazalema, Puerto de las Palomas. 30STF8774. 1200 m. 20-V-80. Hernández Bermejo. COA 9974.

La primera cita para Andalucía Occidental 
aparece en Badolatosa (Sevilla), muy localizada (Infante y Muñoz, 1987). Posteriormente fue encontrada en Baena (Córdoba) sobre margas yesosas (García-Montoya y Muñoz, 1990). Por tanto, esta es la primera cita para la provincia de Cádiz. Se presenta sobre calizas en zona de montaña, con lo que se amplía tanto su área de distribución como su rango altitudinal.

\section{Narcissus tazetta L. subsp. tazetta}

CÓRDOBA: carretera de Medina Azahara, km 2. 30SUG3695. 210 m. 15-IV-97. Lora. COA 24268.

Aparece en un terreno encharcado por afloramiento subterráneo. La única cita en la zona data del siglo XVIII y señala que "se cría con abundancia en sitios húmedos de los campos de Córdoba, como en la Albayda y en el cerro de las Ermitas» (Jordano y Ocaña, 1956). Al ser un taxón que se presentaba en ambientes fuertemente antropizados le tuvo que afectar notablemente el crecimiento demográfico y la roturación de nuevas tierras. Esta localidad, además de ser nueva, confirma la presencia de la especie en el Valle del Guadalquivir.

\section{BIBLIOGRAFÍA}

ALBAREAL, J. y C. ROMERO ZARCO -1993Novedades florísticas para la Campiña y las Sierras Subbéticas sevillanas. Lagascalia 17(1): 190-193. ALCÁNTARA, A.J., A.R. TUDELA, M. CUETO y C. FERNÁNDEZ-LÓPEZ -1990- Compuestas de la provincia de Jaén, III. Blancoana 8: 101-116.

APARICIO, A. -1993- Planes de recuperación de especies vegetales amenazadas en el Parque Natural de la Sierra de Grazalema (CádizMálaga). Acta Bot. Malacitana 18: 199-221.

APARICIO, A. y S. SILVESTRE -1987- Flora del Parque Natural de la Sierra de Grazalema. Junta de Andalucía. A.M.A. Sevilla.

BENAVENTE, A. -1996- Catálogo de fanerógamas del Parque Natural de las Sierras de Cazorla, Segura y las Villas. Taller de Ecología-CODA. Jaén.

BLANCA, G. -1989-Contribución a la flora de la provincia de Jaén. Acta Bot. Malacitana 14: 285-291.

BLANCA, G. -1993- Draba. In: Castroviejo, S. et al. (Eds.), Flora Ibérica. Vol IV. Real Jardín Botánico de Madrid. CSIC.

CUATRECASAS, J. -1929- Estudios sobre la flora y la vegetación del macizo de Mágina. Trab. Mus. Ci. Nat. Barcelona vol. XII.

ESCUDERO, A., S. PAJARÓN y M. COSTA 1989- Elementos térmico-mediterráneos en las inmediaciones del Moncayo. Bot. Complutensis 15: 225-232

FERNÁNDEZ-GALIANO, E. y B. CABEZUDO 1976- Plantas de la reserva biológica de Doñana (Huelva). Lagascalia 6(1): 117-176.

FERNÁNDEZ-GALIANO, E. y V.H. HEYWOOD 1960- Catálogo de plantas de la provincia de Jaén (mitad oriental). Inst. Est. Jiennenses. Jaén.

FERNÁNDEZ-LÓPEZ, C. -1979- Flora y vegetación del Suroeste de la provincia de Jaén. Tesis doctoral. Univ. Granada.

GARCÍA-HERNÁNDEZ, E. y C. FERNÁNDEZLÓPEZ -1986- Malváceas de la provincia de Jaén. Blancoana 4: 153-158.

GARCÍA-MONTOYA, F. y J.M. MUÑOZ -1990Novedades corológicas para la flora de Andalucía Occidental. Lagascalia 16(1): 146-148.

GÓMEZ-CAMPO, C. -1993-Vella. In: Castroviejo, S. et al. (Eds.), Flora Ibérica. Vol IV. Real Jardín Botánico de Madrid. CSIC

HERVIER -1905-Bull. Acad. Int. Geogr. Bot. Le Mans 15: 1-32.

INFANTE, F. y J. MUÑOZ - 1986- Notas breves $n^{\circ}$ 221. Lagascalia 14(1): 160.

JORDANO, D. y M. OCAÑA - 1956- Catálogo del herbario de los botánicos cordobeses Rafael de León y Gávez, Fr. José de Jesús Muñoz Cazalla, Rafael Entrenas y Antonio Cabrera. Anales Inst. Bot. Cavanilles 14: 597-717.

KÜPFER, Ph. -1993- Hormathophylla. In: Castroviejo, S. et al. (Eds.), Flora Ibérica. Vol IV. Real Jardín Botánico de Madrid. CSIC.

LEADLAY, E.A. - 1993- Coincya. In: Castroviejo, S. et al. (Eds.), Flora Ibérica. Vol IV. Real Jardín Botánico de Madrid. CSIC

LÓPEZ-GONZÁLEZ, G. -1990-Arenaria. In: Castroviejo, S. et al. (Eds.), Flora Ibérica. Vol. II. Real Jardín Botánico de Madrid. CSIC.

MEDINA, J.L. y C. FERNÁNDEZ-LÓPEZ - 1988Crucíferas de la provincia de Jaén, II. Blancoana 6: 69-86.

MOLERO, J. -1981- Aportaciones al conocimiento de la flora aragonesa II. Fol. Bot. Misc. 2: 41-48.

MORENO, M. -1983- Iberis grosii Pau: una especie poco conocida de la flora andaluza. Anales Jard. Bot. Madrid 40(1): 53-61. 
PERIS, J.B. y G. STÜBING -1985-Silene oropediorum, una especie nueva para la flora europea. Anales Jard. Bot. Madrid 41(2): 453. RIVAS-MARTÍNEZ, S., M. COSTA, S. CASTROVIEJO y E. VALDÉS -1980- Vegetación de Doñana (Huelva, España). Lazaroa 2: 5-190.

RIVAS-MARTÍNEZ, S., M.E. GARCÍA y A. PENAS -1991- Revisión taxonómica de la sección Aizopsis DC. del género Draba L. en la Península Ibérica. Candollea 46: 439-473.

SCHULZ, O.E. -1957- Cruciferae- Sisymbrieae. In: Engler, A. (Ed.), Das Pflanzenreich, 86. Neudruck.

SAGREDO, R. -1987- Flora de Almería. Inst. Est. Almerienses. Almería.

TALAVERA, S. -1990- Silene L. In: Castroviejo, S. et al. (Eds.). Flora Ibérica. Vol. II. Real Jardín Botánico de Madrid. CSIC.

UBERA, J.L. y B. VALDÉS - 1983- Revisión del género Nepeta (Labiatae) en la Península Ibérica e Islas Baleares. Lagascalia 12(1): 3-80.
VALDÉS, B., S. TALAVERA y E. FERNÁNDEZGALIANO - 1987- Flora vascular de Andalucía occidental. Vol II. Ketres editora. Barcelona. VALLE, F. y C. DÍAZ DE LA GUARDIA - 1987- La Alfaguara y su entorno vegetal. Granada.

VOGT, R. -1991- Die Gattung Leucanthemum Mill. (Compositae-Anthemidae) auf der Iberischen Halbinsel. Monografías del Real Jardín Botánico 10. CSIC.

Aceptado para su publicación en Junio de 1998

Dirección de los autores. A. Lora González: Dpto. de Ciencias y Recursos Agrícolas y Forestales, UCO. Jardín Botánico de Córdoba. Avda. de Linneo s/n. 14004 Córdoba. J.L. Vivero Pol: Jardín Botánico de Córdoba. Avda. de Linneo s/n. 14004 Córdoba.

\title{
58. HYPOCHAERIS ANGUSTIFOLIA (ASTERACEAE): DISTRIBUCIÓN, ECOLOGÍA Y FITOSOCIOLOGÍA
}

\author{
Antonio GALÁN DE MERA y José Alfredo VICENTE ORELLANA
}

Hypochaeris angustifolia (Asteraceae): distribution, ecology and phytosociology.

Palabras clave. Hypochaeris, fitogeografía, Marruecos.

Key Words. Hypochaeris, phytogeography, Morocco.

El género Hypochaeris L. está Mediterránea y América del Sur (Cerbah et al., ámpliamente repartido en el mundo con dos 1995). De todas sus especies, tal vez la menos grandes centros de dispersión, la cuenca conocida es $H$. angustifolia (Litard. \& Maire) Maire, puesto que la mayor parte del material

Este trabajo ha sido realizado con los fondos del proyecto de investigación 12/95 de la Universidad San Pablo-CEU. 